\title{
Low-power cross polarization in fast magic-angle spinning NMR experiments
}

\author{
Adam Lange, Ingo Scholz, Theofanis Manolikas, Matthias Ernst, Beat H. Meier* \\ Physical Chemistry, ETH Zurich, 8093 Zurich, Switzerland
}

\section{A R T I C L E I N F O}

\section{Article history:}

Received 5 October 2008

In final form 26 November 2008

Available online 3 December 2008

\begin{abstract}
A B S T R A C T
We describe a low-power approach for heteronuclear cross-polarization $(\mathrm{CP})$ at high magic-angle spinning (MAS) frequencies. It is based on second-order CP at the $n=0$ Hartmann-Hahn condition. The mechanism for the polarization transfer in the low-power $\mathrm{CP}$ experiment relies on second-order cross terms between homonuclear and heteronuclear dipolar couplings. At a MAS frequency of $65 \mathrm{kHz}$, rf-field amplitudes below $10 \mathrm{kHz}$ are sufficient to efficiently transfer polarization from ${ }^{1} \mathrm{H}$ to ${ }^{13} \mathrm{C}$. The low-rf field requirements of this approach make it well suited for the investigation of proteins and other temperature-sensitive samples without the risk of sample heating and degradation.
\end{abstract}

(c) 2008 Elsevier B.V. All rights reserved.

\section{Introduction}

The development of solid-state magic-angle spinning (MAS) NMR techniques has been stimulated by the availability of increasing MAS frequencies. Presently, spinning frequencies of $70 \mathrm{kHz}$ and more are possible [1-3] and initial worries that the centrifugal forces might degrade the sample quality, in particular for proteins in microcrystalline form, have not proven to be justified. The centrifugal acceleration reaches about $8000000 \mathrm{~g}$ at $70 \mathrm{kHz}$ for a rotor with an inner diameter of $0.8 \mathrm{~mm}$. For biological compounds, where the amount of sample is often limited, small-diameter rotors and fast spinning have opened new opportunities [1]. In addition, higher MAS frequencies move spinning sidebands out of the observed region of the carbon spectrum in high magnetic fields that are essential for structural studies on biomolecules [3-9]. Furthermore, fast MAS allows the use of pulse sequences with lower rf-power requirements because the regime with rf-nutation frequencies significantly below the spinning frequency [2] becomes accessible. In this regime sample heating can be dramatically reduced which is crucial for the investigation of sensitive samples and often allows to shorten the repetition rate of the experiment.

In this communication we concentrate on the cross-polarization (CP) $[10,11]$ step which is a key element in many solid-state NMR experiments. $\mathrm{CP}$ is also used in most experiments on solid proteins, which are often particularly sensitive to heating effects. It requires the simultaneous rf irradiation on two nuclei and the matching of the Hartmann-Hahn condition $\left|\omega_{\mathrm{S}} \pm \omega_{\mathrm{I}}\right|=n \cdot \omega_{\mathrm{r}}$, where $\omega_{\mathrm{I}}$ and $\omega_{\mathrm{S}}$ are the I-spin and S-spin nutation frequencies, respectively, and $\omega_{\mathrm{r}}$ is the MAS frequency [12-15]. The $n=1$ and 2 Hartmann-Hahn conditions dominate the matching profile in the initial rate regime but $\mathrm{CP}$ is also possible at other values of $n[14,15]$. While the

\footnotetext{
* Corresponding author.

E-mail addresses: beme@ethz.ch, beme@nmr.phys.chem.ethz.ch, beat.meier@ gmx.ch (B.H. Meier).
}

Hartmann-Hahn condition fixes only the difference of the rf-field strengths, there are considerations that further constrain the possible values of the individual rf-field amplitudes. Care has to be taken to prevent signal loss due to resonance effects between sample rotation and rf irradiation (e.g. HORROR [16] and rotary-resonance effects [17]) that occur at ratios $\kappa=\omega_{1} / \omega_{\text {r }}$ with $\kappa=1 / 2$ and 1,2 , respectively. For example, it would be possible to use a rf-field amplitude of $165 \mathrm{kHz}$ on ${ }^{1} \mathrm{H}$ (i.e. well above $2^{*} 65 \mathrm{kHz}$ ) and $100 \mathrm{kHz}$ on ${ }^{13} \mathrm{C}$ (i.e. $165 \mathrm{kHz}-65 \mathrm{kHz}$ ) at a MAS frequency of $65 \mathrm{kHz}$.

We concentrate in the following on dipolar cross-polarization using low-amplitude rf irradiation in an effort to minimize sample heating. Sample heating is intrinsically tied to rf irradiation through the electric component of the rf field, although it can be minimized at the sample position by a suitable resonator design, e.g. in so called E-free probes [18-20]. If both amplitudes should be kept below a value of half the spinning frequency, we must exploit the $n=0$ Hartmann-Hahn condition $\left(\omega_{\mathrm{I}}=\omega_{\mathrm{S}}\right)$ where dipolar cross-polarization is only allowed as a second-order cross-polarization (SOCP) process.

Fast spinning and low-power $\mathrm{CP}$ are also of interest in the context of investigating partially deuterated samples in connection with proton detection $[3,21,22]$. Ishii and co-workers have proposed to enhance the sensitivity of solid-state NMR experiments by using paramagnetic metal ions for shortening ${ }^{1} \mathrm{H} T_{1}$ relaxation times $[22,23]$. Here, the reduced recycle delay requires the use of NMR sequences with a reduced power deposition. Low-power cross-polarization could be a building block in such experiments.

We note that low-power cross-polarization intrinsically becomes band selective with respect to the ${ }^{13} \mathrm{C}$ chemical-shift because low-rf fields cannot spin lock the entire range of chemical shifts. For ${ }^{15} \mathrm{~N}$ spectra, the effect is less pronounced because all amide resonances resonate within a relatively small range of chemical shifts. In the context of multidimensional experiments for assignment and structure determination in proteins, this band 
selectivity can also be a desired feature in the spirit of the SPECIFIC CP experiment [24].

\section{Theory and simulations}

At the $n=0$ Hartmann-Hahn condition two different mechanisms can lead to polarization transfer: (i) transfer mediated by the isotropic J-coupling and (ii) transfer due to second-order cross terms between homonuclear and heteronuclear dipolar couplings [25]. In a doubly rotating interaction frame where the $z$ axis is along the spin-lock fields, the J-coupling gives rise to an effective zeroth-order Hamiltonian for an IS two-spin system of

$\bar{H}^{(0)}=\pi \mathrm{J} \frac{1}{2}\left(\mathrm{I}^{+} \mathrm{S}^{-}+\mathrm{I}^{-} \mathrm{S}^{+}\right)$

In proteins, the J-couplings are typically small compared to the dipolar interactions with ${ }^{1} \mathrm{~J}_{\mathrm{CH}} \approx 150 \mathrm{~Hz}$ although in other systems, the J-transfer can dominate [26]. The second-order cross-terms between a homonuclear and a heteronuclear dipolar coupling in an $\mathrm{I}_{2} \mathrm{~S}$ three-spin system are given by [25]

$\bar{H}^{(2)}=\omega_{\mathrm{S} 1}\left(\mathrm{~S}^{-} \mathrm{I}_{1}^{+} \mathrm{I}_{2 z}+\mathrm{S}^{+} \mathrm{I}_{1}^{-} \mathrm{I}_{2 z}\right)+\omega_{\mathrm{S} 2}\left(\mathrm{~S}^{-} \mathrm{I}_{2}^{+} \mathrm{I}_{1 z}+\mathrm{S}^{+} \mathrm{I}_{2}^{-} \mathrm{I}_{1 z}\right)$

where $\omega_{\mathrm{S} 1}$ and $\omega_{\mathrm{S} 2}$ depend on the orientation and magnitude of all three dipolar couplings as well as on the spinning frequency and the rf-field amplitudes. Detailed expressions for these effective coupling constants were derived in Ref. [25] and can be found in the Supplementary material. It should be noted, that two I spins are necessary for this type of Hartmann-Hahn transfer. For an isolated two-spin system (e.g. an $\mathrm{N}-\mathrm{H}$ in a highly deuterated protein), no dipolar transfer is expected.

The theoretical results are supported by numerical simulations of low-power CP build-up curves carried out with the GAMMA [27] spin-simulation environment. We considered a single carbon spin connected to two protons, similar to the situation in a $\mathrm{CH}_{2}$ group. The $\left({ }^{1} \mathrm{H}-{ }^{1} \mathrm{H}\right)$ and $\left({ }^{13} \mathrm{C}-{ }^{1} \mathrm{H}\right)$ distances were assumed to be $1.8 \AA$ and $1.1 \AA$, respectively, and a $\left({ }^{1} \mathrm{H}-{ }^{13} \mathrm{C}\right) \mathrm{J}$-coupling of $155 \mathrm{~Hz}$ was used. Spin-lock fields with an amplitude of $10 \mathrm{kHz}$ were applied to ${ }^{1} \mathrm{H}$ and ${ }^{13} \mathrm{C}$ at a MAS frequency of $65 \mathrm{kHz}$. The initial density operator was set to spin-locked proton polarization, $\hat{\sigma}(0)=\hat{\mathrm{I}}_{1 z}+\hat{\mathrm{I}}_{2 z}$.

The dipolar spectrum corresponding to the time evolution of $\sigma(0)$ under the Hamiltonian of Eq. (2) is displayed in Fig. 1. Only dipolar interactions were considered in this simulation. The effective dipolar coupling is described by an effective coupling constant

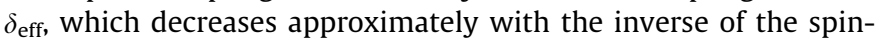
ning frequency. At $65 \mathrm{kHz}$ MAS frequency the effective coupling constants are approximately $2 \mathrm{kHz}$ and, therefore, still considerably larger than the ${ }^{1} \mathrm{~J}_{\mathrm{CH}}$-coupling constant.

The plotted signal intensities in Fig. 2 are the expectation values of $\hat{\mathrm{S}}_{z}, \hat{\mathrm{I}}_{1 z}$, and $\hat{\mathrm{I}}_{2 z}$, respectively. In total six different combinations of dipolar and J-couplings were simulated for the $\mathrm{CP}$ experiment in the $\mathrm{CH}_{2}$ group. The results are shown in Fig. 2 together with a pictorial representation of the considered coupling network. The simulations show that the presence of a single $\left({ }^{1} \mathrm{H}-{ }^{13} \mathrm{C}\right)$ and a $\left({ }^{1} \mathrm{H}-{ }^{1} \mathrm{H}\right)$ dipolar coupling lead to transfer from both protons to the carbon spin (Fig. 2a). On the other hand, the presence of two heteronuclear $\left({ }^{1} \mathrm{H}-{ }^{13} \mathrm{C}\right)$ dipolar couplings only does not give significant transfer (Fig. 2b). In a system with all three dipolar couplings present (Fig. 2c), the transfer efficiency compared to direct excitation is about 2.3. The situation does not change much when also heteronuclear $\left({ }^{1} \mathrm{H}-{ }^{13} \mathrm{C}\right) \mathrm{J}$-couplings are included (Fig. 2e). While lowpower CP is not possible for an isolated dipolar coupled $\left({ }^{1} \mathrm{H}-{ }^{13} \mathrm{C}\right)$ spin system (Fig. 2d) the inclusion of a J-coupling (Fig. 2f) leads to a characteristic oscillatory transfer. The timescale of the J-coupling mediated polarization transfer is about one order of

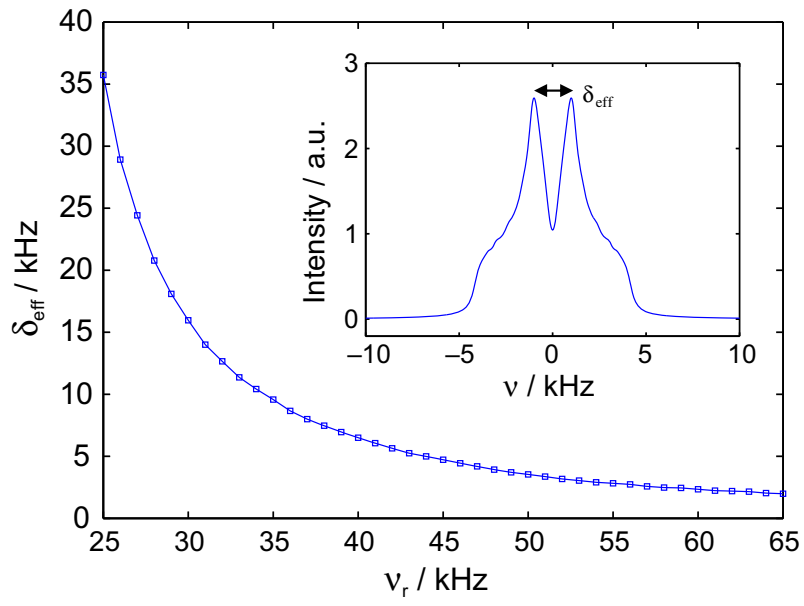

Fig. 1. Numerical simulation of effective dipolar coupling pattern under low-power $\mathrm{CP}$ conditions based on the effective Hamiltonian in a $\mathrm{CH}_{2}$-spin system as a function of the spinning frequency. The effective coupling constant $\delta_{\text {eff }}$ was measured from the distance between the two maxima in the observed powder pattern. The inset shows as an example the simulated powder pattern obtained from the Fourier transform of the time domain signal at $50 \mathrm{kHz}$. For all simulations both rf-field amplitudes were set to $10 \mathrm{kHz}$. Only dipolar couplings were taken into account. For the powder averaging 1154 orientations were considered.

magnitude smaller compared to the transfer mediated by the second-order dipolar terms (Fig. 2a, c, and e).

Having established the mechanism for polarization transfer and the condition on the difference of the two rf-field strengths, we now turn to the additional considerations regarding the spin-lock fields involved. Here, the emphasis is on avoiding recoupling conditions that lead to a fast loss of the spin-locked magnetization. These conditions are clearly seen in the experimental spin-lock spectrum of Fig. 3. Details are given in the Experimental part and in the present context we only note the fast magnetization decay at ratios $\kappa=\omega_{1} / \omega_{\mathrm{r}}$ of $\kappa=1 / 4,1 / 3,1 / 2,1,3 / 2$ and 2 (marked by asterisks). A key point for the low-power $\mathrm{CP}$ experiment is the observation that at high MAS frequencies ( $>45 \mathrm{kHz}$ for a typical organic solid) efficient spin locking is possible not only at rf fields above the resonance conditions described before but also below.

In the following we describe these undesired resonance conditions considering a spin lock on the I spins in an $\mathrm{I}_{\mathrm{N}} \mathrm{S}$-spin system. While resonance effects that occur at ratios $\kappa=\omega_{1} / \omega_{\mathrm{r}}$ with $\kappa=1 / 2,1$, and 2 are well described in terms of zeroth-order perturbation theory in a Floquet description, effects at $\kappa=1 / 4,1 / 3$, and $3 / 2$ can only be understood in terms of higher-order perturbation theory within the Floquet framework [25].

At the HORROR condition $(\kappa=1 / 2)$ homonuclear dipolar couplings of the I spins are recoupled with an effective (doublequantum) Hamiltonian

$\bar{H}^{(0)}=\frac{3}{4} \sum_{m<n}\left(\omega_{m n}^{(-1)} \mathbf{I}_{m}^{+} \mathbf{I}_{n}^{+}+\omega_{m n}^{(+1)} \mathbf{I}_{m}^{-} \mathbf{I}_{n}^{-}\right)$

Matching the $\kappa=1$ rotary-resonance condition leads to a recoupling of the I-spin chemical-shift (CSA) tensors, heteronuclear dipolar couplings, and I-spin homonuclear dipolar couplings, giving rise to a Hamiltonian of

$$
\begin{aligned}
\bar{H}^{(0)}= & \frac{3}{4} \sum_{m<n}\left(\omega_{m n}^{(-2)} \mathrm{I}_{m}^{+} \mathrm{I}_{n}^{+}+\omega_{m n}^{(+2)} \mathrm{I}_{m}^{-} \mathrm{I}_{n}^{-}\right)-\frac{1}{2} \sum_{m}\left(\omega_{m}^{(-1)} \mathrm{I}_{m}^{+}+\omega_{m}^{(+1)} \mathrm{I}_{m}^{-}\right. \\
& \left.+\omega_{m \mathrm{~s}}^{(-1)} 2 \mathrm{~S}_{z} \mathrm{I}_{m}^{+}+\omega_{m \mathrm{~S}}^{(+1)} 2 \mathrm{~S}_{z} \mathrm{I}_{m}^{-}\right)
\end{aligned}
$$

At the $\kappa=2$ rotary-resonance condition, only the I-spin CSA tensors and the heteronuclear couplings are recoupled 

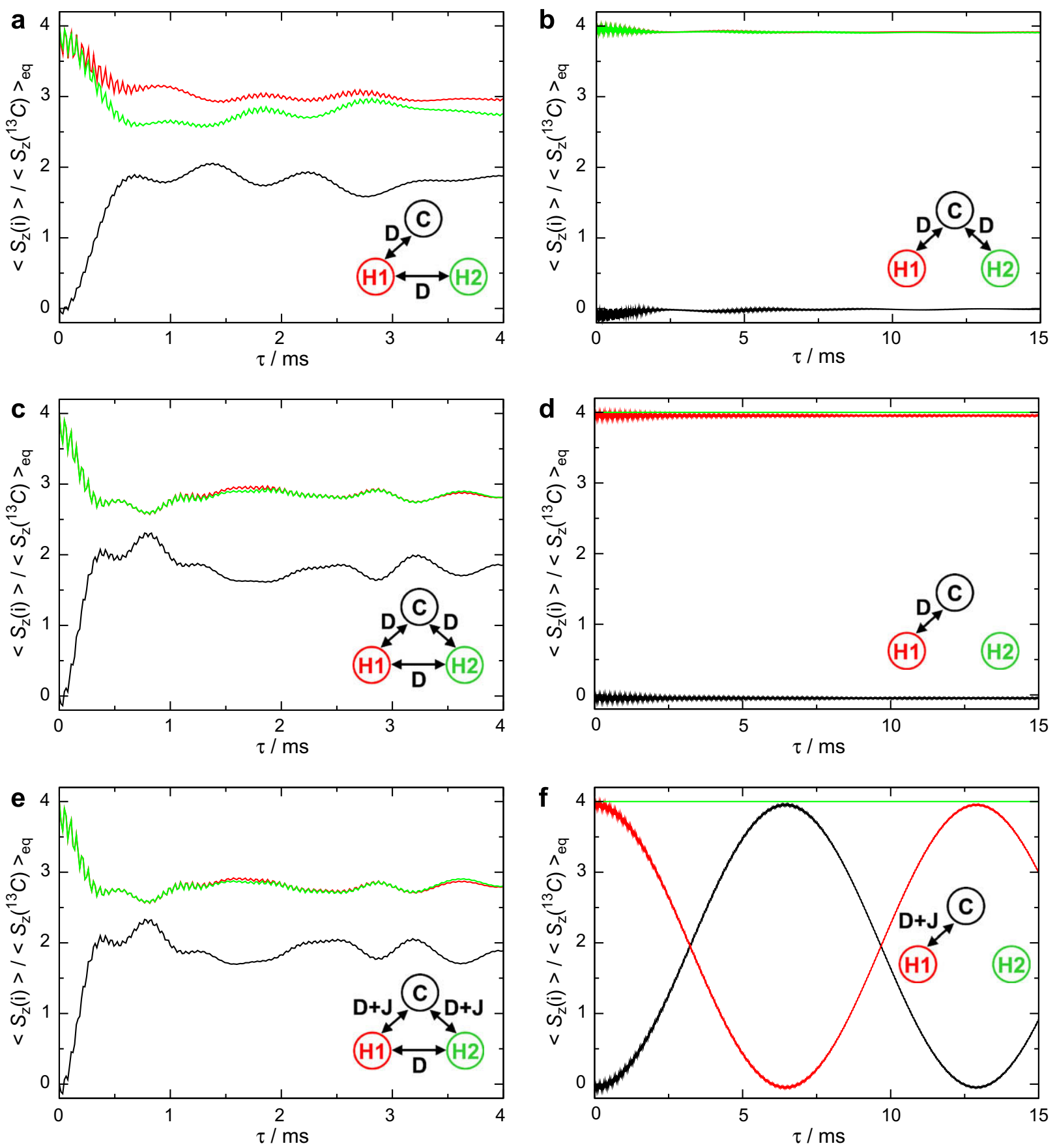

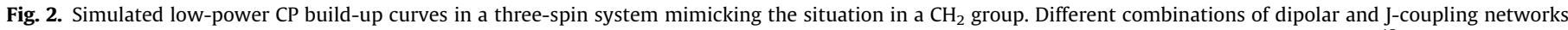

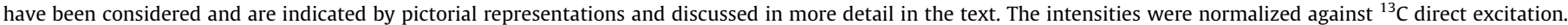
Two-hundred orientations were considered for the powder averaging.

$\bar{H}^{(0)}=-\frac{1}{2} \sum_{m}\left(\omega_{m}^{(-2)} \mathrm{I}_{m}^{+}+\omega_{m}^{(+2)} \mathrm{I}_{m}^{-}+\omega_{m \mathrm{~S}}^{(-2)} 2 \mathrm{~S}_{z} \mathrm{I}_{m}^{+}+\omega_{m \mathrm{~S}}^{(+2)} 2 \mathrm{~S}_{z} \mathrm{I}_{m}^{-}\right)$

The coefficients $\omega_{m S}^{(n)}, \omega_{m n}^{(n)}$, and $\omega_{m}^{(n)}$ involved in the zeroth-order recoupling conditions given above are the Fourier coefficients of the time-dependent interactions as defined in Ref. [28] and the Supplementary material.

At the resonance condition $\kappa=3 / 2$, homonuclear dipolar couplings are reintroduced. The effective Hamiltonian as derived by second-order perturbation theory is

$\bar{H}^{(2)}=-\sum_{m<n<0} \omega_{m n o}^{-} 2 \mathrm{I}_{m z} \mathrm{I}_{n}^{-} \mathrm{I}_{o}^{-}-\sum_{m<n<o} \omega_{m n o}^{+} 2 \mathrm{I}_{m z} \mathrm{I}_{n}^{+} \mathrm{I}_{o}^{+}$
Third-order recoupling conditions can be observed at $\kappa=1 / 3$

$$
\begin{aligned}
\bar{H}^{(3)}= & \sum_{m<n<0}\left(\omega_{\text {Smno }}^{-} 2 \mathrm{~S}_{z} \mathrm{I}_{m}^{-} \mathrm{I}_{n}^{-} \mathrm{I}_{o}^{-}+\omega_{\text {Smno }}^{+} 2 \mathrm{~S}_{z} \mathrm{I}_{m}^{+} \mathrm{I}_{n}^{+} \mathrm{I}_{o}^{+}\right) \\
& +\sum_{m<n<0}\left(\omega_{m n o}^{-} \mathrm{I}_{m}^{-} \mathrm{I}_{n}^{-} \mathrm{I}_{o}^{-}+\omega_{m n o}^{+} \mathrm{I}_{m}^{+} \mathrm{I}_{n}^{+} \mathrm{I}_{o}^{+}\right)
\end{aligned}
$$

and also a previously not observed pure homonuclear dipolar recoupling condition at $\kappa=1 / 4$

$\bar{H}^{(3)}=\sum_{m<n<0<p}\left(\omega_{m n o p}^{-} \mathrm{I}_{m}^{-} \mathrm{I}_{n}^{-} \mathrm{I}_{o}^{-} \mathrm{I}_{p}^{-}+\omega_{m n o p}^{+} \mathrm{I}_{m}^{+} \mathrm{I}_{n}^{+} \mathrm{I}_{o}^{+} \mathrm{I}_{p}^{+}\right)$ 


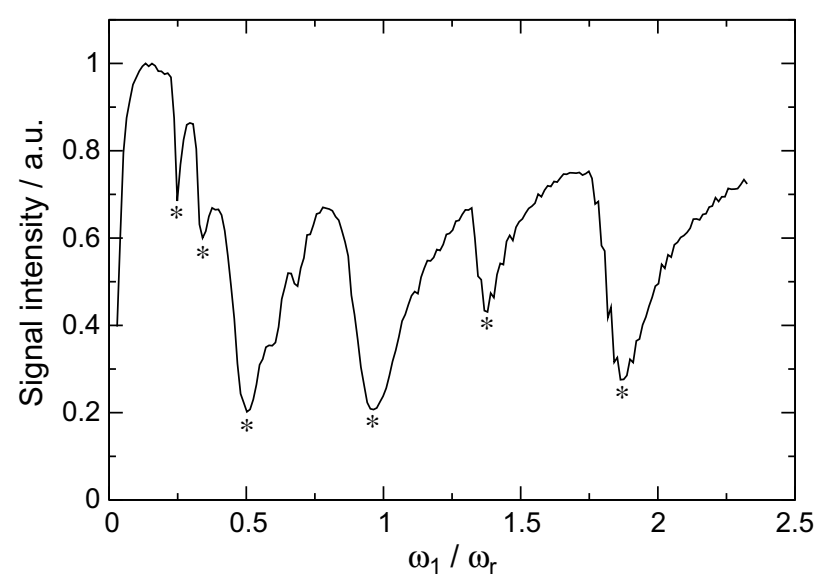

Fig. 3. ${ }^{1} \mathrm{H}$ spin-lock efficiency as a function of the applied rf-field amplitude indirectly detected by cross polarization to the $\mathrm{C}^{\alpha}$ resonance of glycine ethyl ester. Resonance conditions as described in the text are labeled with asterisks.

Note that for the last two of the undesired conditions at least 3 (in the case of $\kappa=1 / 3)$ and $4(\kappa=1 / 4)$ dipolar coupled I spins are required.

\section{Materials and methods}

Uniformly $\left[{ }^{13} \mathrm{C},{ }^{15} \mathrm{~N}\right]$ labeled microcrystalline ubiquitin was prepared as described before [29]. One-dimensional NMR experiments were performed on a $20 \mathrm{~T}$ (corresponding ${ }^{1} \mathrm{H}$-resonance frequency: $850 \mathrm{MHz}$ ) wide-bore magnet (Bruker Biospin, Germany) equipped with a $1.3 \mathrm{~mm}$ double-resonance MAS probe. The MAS frequency was set to $65 \mathrm{kHz}$. All experiments were performed at cooling gas temperatures of $298 \mathrm{~K}\left(1,2-{ }^{13} \mathrm{C}\right.$ glycine ethyl ester) and $235 \mathrm{~K}$ (ubiquitin), respectively. For glycine ethyl ester continuous-wave decoupling with an rf-field amplitude of $25-30 \mathrm{kHz}$ was used. On ubiquitin low-power XiX decoupling [30] with an rf-field amplitude of $15-20 \mathrm{kHz}$ was performed.

\section{Results and discussion}

To identify suitable ${ }^{1} \mathrm{H}$ spin-lock conditions for cross polarization, the ${ }^{1} \mathrm{H}$ spin-lock efficiency was measured as a function of the spin-lock amplitude, $\omega_{1}$, at a MAS frequency of $65 \mathrm{kHz}$ indirectly via the $\mathrm{C}^{\alpha}$ resonance of our test system, glycine ethyl ester, using a spin-lock time of $2.5 \mathrm{~ms}$ and subsequent cross polarization at a fixed matching condition. The resulting signal intensity as a function of $\omega_{1}$ is depicted in Fig. 3 and shows the resonance conditions discussed in the Theory section and which lead to a fast decay of the spin-locked magnetization. In the plot these conditions are marked with asterisks. The most pronounced effects occur at ratios $\kappa=\omega_{1} / \omega_{\mathrm{r}}$ with $\kappa$ equal to $1 / 2,1$, and 2 which appear in zerothorder perturbation theory. Weaker resonances are observed at values of $\kappa$ equal to $1 / 4,1 / 3$, and $3 / 2$. These conditions can be understood in terms of higher-order perturbation theory. The best spin-lock efficiency occurred for a rf-field amplitude of $9.4 \mathrm{kHz}$,
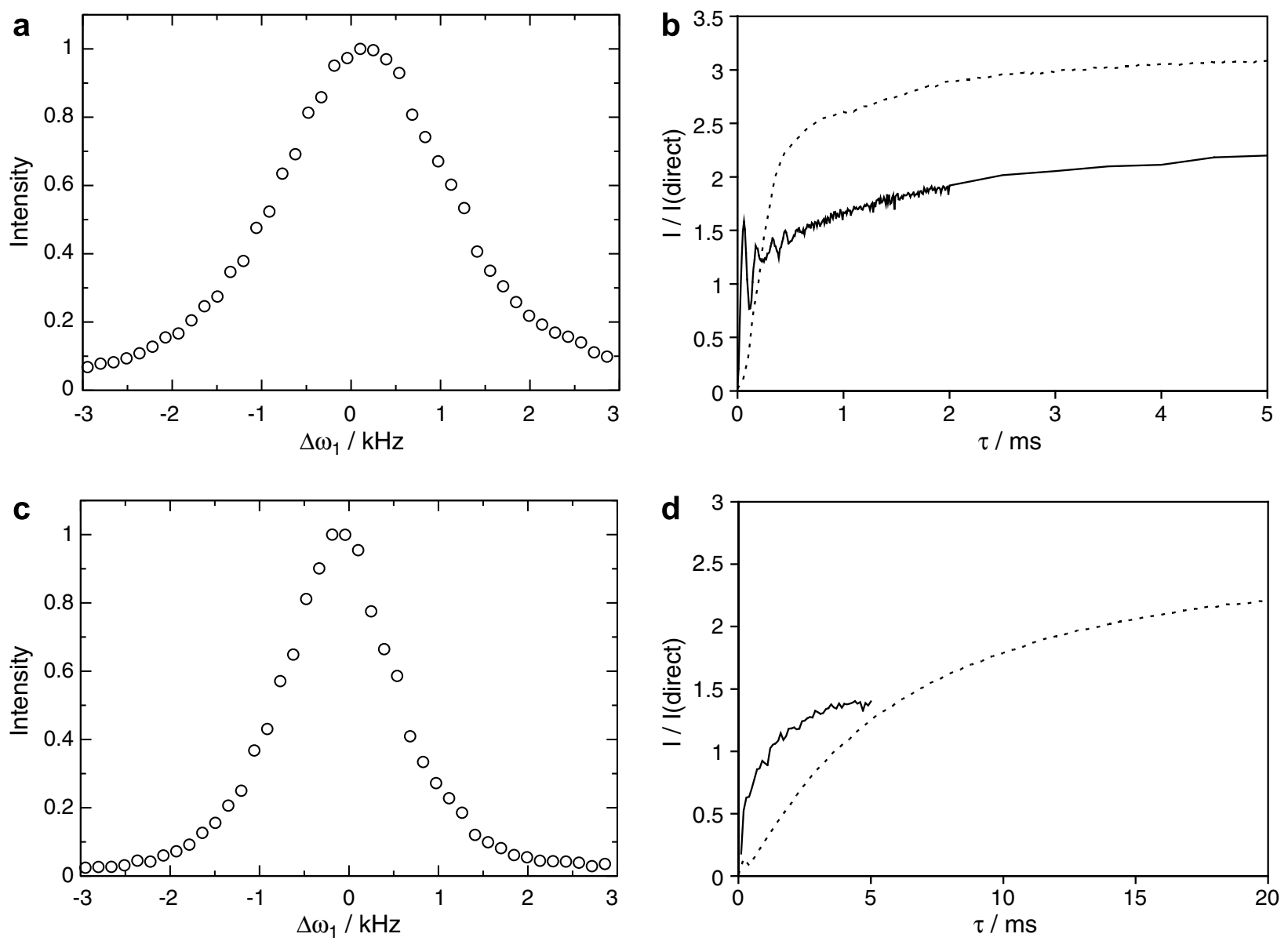

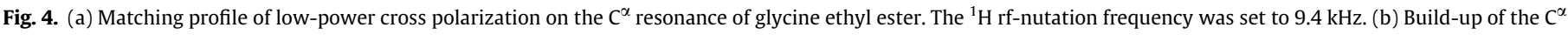

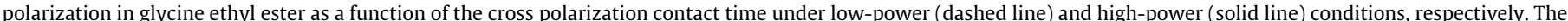

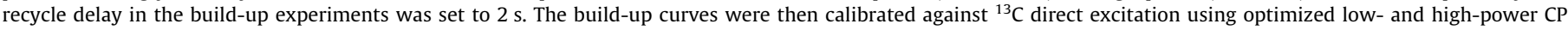
spectra with a $10 \mathrm{~s}$ recycle delay as a reference (c) as in panel a but for $\mathrm{C}^{\prime}$. (d) as in panel b but for $\mathrm{C}^{\prime}$. 
well below all resonance conditions. In principle, going to very high spin-lock fields should also allow efficient spin locking, but the required spin-lock fields lead to strong sample heating.

Next, low-power CP to the $\mathrm{C}^{\alpha}$ or the $\mathrm{C}^{\prime}$ resonance of $1,2-{ }^{13} \mathrm{C}$ glycine ethyl ester was optimized. On the proton channel, an onresonance low-power spin-lock field with a rf-field amplitude of $9.4 \mathrm{kHz}$ was applied. The matching condition for the ${ }^{13} \mathrm{C}$ rf field is shown in Fig. 4a for the $\mathrm{C}^{\alpha}$ resonance and in Fig. 4c for the $\mathrm{C}^{\prime}$ resonance. In both cases, the optimum ${ }^{13} \mathrm{C}$ field is close to the theoretical $n=0$ Hartmann-Hahn condition with rf amplitudes of $9.4 \mathrm{kHz}$ on both channels. The build-up of signal intensity with increasing SOCP contact time is shown as a dashed line in Fig. 4b for the $\mathrm{C}^{\alpha}$ and in Fig. $4 \mathrm{~d}$ for the $\mathrm{C}^{\prime}$ resonance. While the $\mathrm{C}^{\alpha}$ signal builds up within the first $2 \mathrm{~ms}$, the build-up of the $\mathrm{C}^{\prime}$ signal is considerably slower and takes approximately an order of magnitude longer. For comparison we have also recorded high-power CP spectra at the $n=1$ Hartmann-Hahn condition with rf-field amplitudes of $165 \mathrm{kHz}$ on ${ }^{1} \mathrm{H}$ and $100 \mathrm{kHz}$ on ${ }^{13} \mathrm{C}$ at a MAS frequency of $65 \mathrm{kHz}$. Under these conditions the $\mathrm{CP}$ build-up is considerably faster because at the $n=1$ and 2 sideband CP conditions, polarization transfer appears as a zeroth-order effect. For $\mathrm{C}^{\alpha}$ a first transient maximum is observed at $60 \mu$ s and the signal slowly increases up to $5 \mathrm{~ms}$ (see Fig. $4 \mathrm{~b}$ solid line). For $\mathrm{C}^{\prime}$ the maximum transfer is reached after $5 \mathrm{~ms}$ (see Fig. $4 \mathrm{~d}$ solid line). Nevertheless, the maximum transfer is higher for low-power SOCP.

In principle, at the $n=0$ Hartmann-Hahn condition polarization transfer via J-couplings is also possible and will, therefore, also contribute to low-power CP [26]. However, the strengths of the relevant interactions in $\mathrm{CH}$-spin systems, as shown in the theoretical calculations and as seen from the experimental build-up rates suggest that J-couplings are not the dominant transfer mechanism and that, indeed, mostly cross terms between $\left({ }^{1} \mathrm{H}-{ }^{1} \mathrm{H}\right)$ and $\left({ }^{1} \mathrm{H}-{ }^{13} \mathrm{C}\right)$ dipolar couplings are responsible.

In order to determine the bandwidth of the experiment, we have measured the signal intensity of the $C^{\alpha}$ resonance of glycine ethyl ester as a function of the ${ }^{13} \mathrm{C}$ chemical-shift offset during low-power $\mathrm{CP}$ with experimental conditions as above. A plot of the data can be found in Fig. 1 of the Supplementary material. The bandwidth is approximately $9 \mathrm{kHz}$ (FWHH) which is in agreement with a spin-lock field of $9.4 \mathrm{kHz}$.

In order to estimate the efficiency of SOCP quantitatively, we compared optimized low-power CP spectra (CP contact times of $10 \mathrm{~ms}$ for $\mathrm{C}^{\alpha}$ and $40 \mathrm{~ms}$ for $\mathrm{C}^{\prime}$, respectively, were used with $10 \mathrm{~s}$ recycling delays) with direct ${ }^{13} \mathrm{C}$ excitation (recycle delay: $1200 \mathrm{~s}$ ). In the case of $\mathrm{C}^{\alpha}$ an enhancement factor of $\sim 3.1$ was observed. No signal was transferred to the $\mathrm{C}^{\prime}$ resonance. For selective transfer to the $C^{\prime}$ resonance we measured a factor of $\sim 2.4$. Similarly, we estimated the efficiency of high-power CP as described above at a contact time of $5 \mathrm{~ms}$ and found values of 2.2 for $\mathrm{C} \alpha$ and 1.4 for $C^{\prime}$. In the thermodynamic limit an enhancement of 3.27 is predicted for high-power $\mathrm{CP}$ considering nine protons and two labeled carbons in glycine ethyl ester. For narrow-banded low-power CP nine protons and one labeled carbon are considered, leading to a theoretical enhancement of 3.60.

Finally, low-power CP spectra of uniformly $\left[{ }^{13} \mathrm{C},{ }^{15} \mathrm{~N}\right]$ labeled microcrystalline ubiquitin (1-2 mg) at $65 \mathrm{kHz}$ MAS spinning and experimental conditions as above ( $\mathrm{CP}$ contact time: $2.1 \mathrm{~ms}$ ) were recorded. Fig. 5b shows such a spectrum where the carrier frequency was set to the $\mathrm{C}^{\alpha}$ region. For comparison, a directly excited ${ }^{13} \mathrm{C}$ spectrum (recycle delay: $300 \mathrm{~s}$ ) is shown in Fig. 5a. The number of scans in both spectra was 16 . The measured enhancement factor for the $C^{\alpha}$ region was $\sim 1.5$. Several reasons could be responsible for the smaller enhancement factor compared to glycine ethyl ester: firstly, the highly hydrated protein sample has a significantly shorter $T_{1 \rho}$. For $T_{1 \rho}\left({ }^{1} \mathrm{H}\right)$ at a rf-field amplitude of $9 \mathrm{kHz}$ we found values of $\sim 5 \mathrm{~ms}$ for ubiquitin and $\sim 700 \mathrm{~ms}$ for glycine ethyl ester.

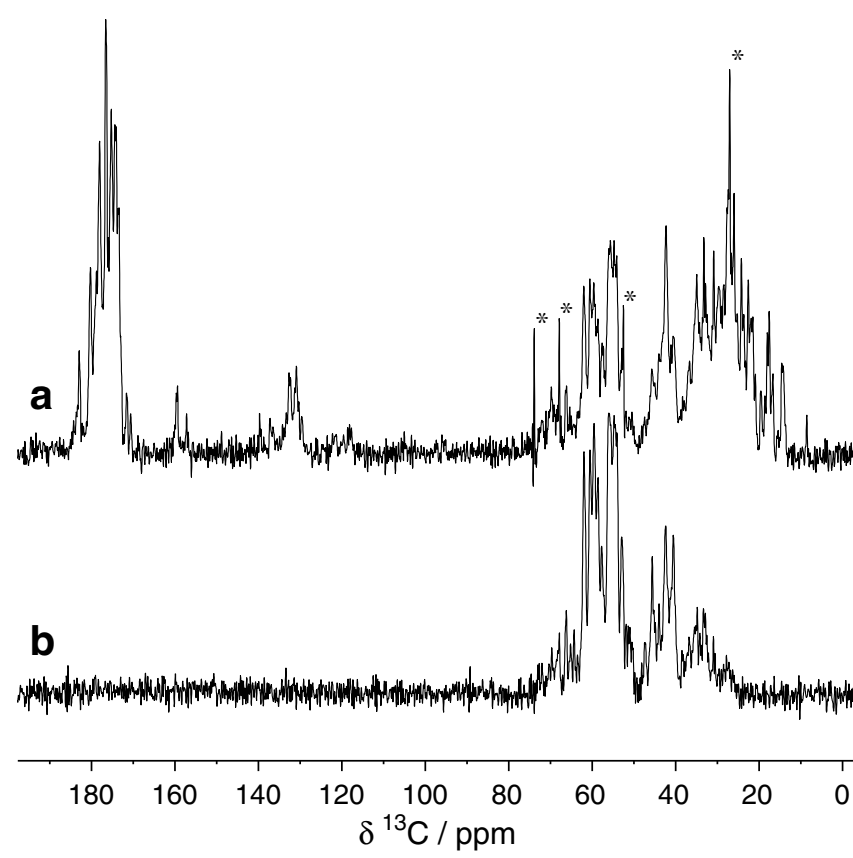

Fig. 5. (a) One-pulse excitation ${ }^{13} \mathrm{C}$ spectrum of $1-2 \mathrm{mg}$ ubiquitin (16 scans). Resonance lines due to the precipitant, MPD are indicated by asterisks (b) Lowpower cross-polarization spectrum of the same sample with the same number of scans. The ${ }^{13} \mathrm{C}$ carrier frequency was set to the $\mathrm{C}^{\alpha}$ region of the spectrum. The $\mathrm{C}^{\mathrm{a}}$ region is very similar but at higher intensity.

Secondly, the ratio of protons to carbons that determines the maximum transfer efficiency is smaller leading to an expected value of 3.49 in the thermodynamic limit (considering that all 524 protons in ubiquitin transfer to $76 \mathrm{C}^{\alpha} \mathrm{s}$ ). In the case of high-power $\mathrm{CP}$ an enhancement of 2.32 is expected (considering 524 protons and 378 carbons).

Nevertheless, since the biggest contribution to the signal enhancement under CP comes from the increased number of scans per unit time, an enhancement factor of 1.5 is still considered efficient. Attempts to obtain a more efficient high-power CP failed. With rf amplitudes of $165 \mathrm{kHz}$ on ${ }^{1} \mathrm{H}$ and $100 \mathrm{kHz}$ on ${ }^{13} \mathrm{C}$ an enhancement factor of 1.1 was found (CP contact time: $2 \mathrm{~ms}$ ).

\section{Conclusions}

We have described efficient band-selective heteronuclear polarization transfer at high magic-angle spinning (MAS) frequencies using the $n=0$ Hartmann-Hahn cross-polarization condition with rf-field amplitudes matched at around $10 \mathrm{kHz}$ for $65 \mathrm{kHz}$ MAS. The SOCP mechanism relies on second-order cross terms between the dipolar $\left({ }^{1} \mathrm{H}-{ }^{1} \mathrm{H}\right)$ and $\left({ }^{1} \mathrm{H}-{ }^{13} \mathrm{C}\right)$ couplings. SOCP spectra of microcrystalline ubiquitin with $\mathrm{CP}$ at rf-field amplitudes of $\sim 9 \mathrm{kHz}$ and decoupling at a rf-field amplitude of $15-20 \mathrm{kHz}$ were recorded. Because the bandwidth of the low-power CP is obviously too small for the whole spectral range of ${ }^{13} \mathrm{C}$, only selected regions can be excited. In some situations, this may be a limitation of low-power $\mathrm{CP}$, in other situations it can be an advantage because magnetization in multidimensional experiments can be channeled to desired pathways [24].

The efficiency of the SOCP experiment turned out to be better than the efficiency in high-power CP experiments with rf-field amplitudes up to $170 \mathrm{kHz}$. It is of course also possible to obtain low-power cross polarization at the sideband Hartmann-Hahn conditions [15] but one of the applied fields must then be higher than half of the spinning frequency. It is a practical advantage of the $n=0 \mathrm{CP}$ condition, that it is less sensitive to rf-inhomogeneity 
and thermal amplifier drifts than the sideband conditions. An alternative approach to polarization transfer at high MAS, in particular for partially deuterated samples, would be INEPT experiments $[31,32]$ which use J-transfer and request long $T_{2}$ times.

\section{Acknowledgments}

We thank Herbert Zimmermann from the MPI für medizinische Forschung in Heidelberg for the synthesis of the selectively labeled glycine ethyl ester sample. Financial support by the ETH Zurich through the TH-Grant System and by the Swiss National Science Foundation is gratefully acknowledged. A.L. thanks the European Molecular Biology Organization (EMBO) for a fellowship.

\section{Note added in proof}

Low power first-order cross polarization for proteins is also discussed in a paper that just appeared on the web: S. Laage, A. Marchetti, J. Sein, R. Pierattelli, J-J. Sass, S. Grzesiek, A. Lesage, G. Pintacuda, L. Emsley J. Am. Chem. Soc., ASAP article.

\section{Appendix A. Supplementary material}

Supplementary data associated with this article can be found, in the online version, at doi:10.1016/j.cplett.2008.11.089.

\section{References}

[1] M. Ernst, A. Detken, A. Bockmann, B.H. Meier, J. Am. Chem. Soc. 125 (2003) 15807.

[2] M. Ernst, M.A. Meier, T. Tuherm, A. Samoson, B.H. Meier, J. Am. Chem. Soc. 126 (2004) 4764.
[3] D.H. Zhou, G. Shah, M. Cormos, C. Mullen, D. Sandoz, C.M. Rienstra, J. Am. Chem. Soc. 129 (2007) 11791.

[4] F. Castellani, B. Van Rossum, A. Diehl, M. Schubert, K. Rehbein, H. Oschkinat, Nature 420 (2002) 98.

[5] A. Lange, S. Becker, K. Seidel, K. Giller, O. Pongs, M. Baldus, Angew Chem. Int. Ed. 44 (2005) 2089.

[6] A. Lange, K. Giller, S. Hornig, M.F. Martin-Eauclaire, O. Pongs, S. Becker, M. Baldus, Nature 440 (2006) 959.

[7] T. Manolikas, T. Herrmann, B.H. Meier, J. Am. Chem. Soc. 130 (2008) 3959.

[8] C. Wasmer, A. Lange, H. Van Melckebeke, A.B. Siemer, R. Riek, B.H. Meier, Science 319 (2008) 1523

[9] S.G. Zech, A.J. Wand, A.E. Mcdermott, J. Am. Chem. Soc. 127 (2005) 8618.

[10] S.R. Hartmann, E.L. Hahn, Phys. Rev. 128 (1962) 2042.

[11] A. Pines, M.G. Gibby, J.S. Waugh, J. Chem. Phys. 59 (1973) 569.

[12] E. Stejskal, J. Schaefer, J. Waugh, J. Magn. Reson. 28 (1977) 105.

[13] M. Sardashti, G. Maciel, J. Magn. Reson. 72 (1987) 467.

[14] S. Hediger, B.H. Meier, R. Ernst, Chem. Phys. Lett. 213 (1993) 627.

[15] B.H. Meier, Chem. Phys. Lett. 188 (1992) 201.

[16] N. Nielsen, H. Bildsoe, H. Jakobsen, M. Levitt, J. Chem. Phys. 101 (1994) 1805.

[17] T. Oas, R. Griffin, M. Levitt, J. Chem. Phys. 89 (1988) 692.

[18] J.A. Stringer et al., J. Magn. Reson. 173 (2005) 40.

[19] B. Dillmann, K. Elbayed, H. Zeiger, M. Weingertner, M. Plotto, F. Engelke, J. Magn. Reson. 187 (2007) 10.

[20] A. Krahn, U. Priller, L. Emsley, F. Engelke, J. Magn. Reson. 191 (2008) 78

[21] R. Linser, V. Chevelkov, A. Diehl, B. Reif, J. Magn. Reson. 189 (2007) 209.

[22] N.P. Wickramasinghe, M. Kotecha, A. Samoson, J. Past, Y. Ishii, J. Magn. Reson. 184 (2007) 350.

[23] N.P. Wickramasinghe, M. Shaibat, Y. Ishii, J. Am. Chem. Soc. 127 (2005) 5796.

[24] M. Baldus, A. Petkova, J. Herzfeld, R. Griffin, Mol. Phys. 95 (1998) 1197.

[25] I. Scholz, B.H. Meier, M. Ernst, J. Chem. Phys. 127 (2007) 204504.

[26] A. Verhoeven, R. Verel, B.H. Meier, Chem. Phys. Lett. 266 (1997) 465.

[27] S. Smith, T. Levante, B.H. Meier, R. Ernst, J. Magn. Reson. Ser. A 106 (1994) 75

[28] M. Ernst, A. Samoson, B.H. Meier, J. Chem. Phys. 123 (2005) 064102.

[29] M. Schubert, T. Manolikas, M. Rogowski, B.H. Meier, J. Biomol. NMR 35 (2006) 167.

[30] M. Ernst, A. Samoson, B.H. Meier, J. Magn. Reson. 163 (2003) 332.

[31] G. Morris, R. Freeman, J. Am. Chem. Soc. 101 (1979) 760.

[32] B. Elena, A. Lesage, S. Steuernagel, A. Bockmann, L. Emsley, J. Am. Chem. Soc. 127 (2005) 17296. 